\title{
High-Dose Intra-Dermal Hepatitis B Vaccine in a Liver Transplant Patient Who Failed Prior Intramuscular Vaccination: A Brief Case Report
}

\author{
Sonu Dhillon ${ }^{1 *}$, Kartik Sampath ${ }^{2}$, Christopher M. Moore ${ }^{2}$, Anil Dosanjh ${ }^{3}$, David H. Van Thiel ${ }^{2}$ \\ ${ }^{1}$ Division of Gastroenterology and Hepatology, Saint Francis Medical Center, Peoria, USA; ${ }^{2}$ Divison of Digestive Diseases, Rush \\ University Medical Center, Chicago, USA; ${ }^{3}$ University of California, Davis, USA. \\ Email: * sonu.dhillon@osfhealthcare.org
}

Received December $24^{\text {th }}, 2012$; revised January $25^{\text {th }}, 2013$; accepted February $3^{\text {rd }}, 2013$

\begin{abstract}
Hepatitis B virus (HBV) chronic infection represents a significant cause of morbidity and mortality worldwide. While traditional intramuscular (IM) HBV vaccination is an excellent method for robust and sustained seroconversion in healthy individuals, its efficacy in chronic liver disease is sub-optimal and scant data exists in the post-liver transplant state. Importantly, HBV complications are even more severe in these same immunocompromised populations. Intra-dermal (ID) vaccination has shown initial promise as a successful alternative to achieving HBV seroconversion in patients refractory to standard vaccination protocols. Herein is a case report of a 61 year-old female who underwent liver transplantation for chronic HBV infection and achieved HBsAg seroconversion with a robust HSsAb titer with ID vaccination after having failed both standard and double dose IM vaccination.
\end{abstract}

Keywords: Hepatitis B Virus; Intra-Dermal Vaccination; Orthotopic Liver Transplant

\section{Introduction}

Hepatitis B virus (HBV) infection is a global health problem with over two billion people with serologic evidence of disease exposure and 360 million who are chronic carriers $[1,2]$. One million deaths can be attributed to development of decompensated cirrhosis and hepatocellular carcinoma in this population. In this patient population orthotopic liver transplantation (OLT) is the primary therapeutic modality for prolonged survival. While initial survival results with OLT in such individuals were discouraging due to rapid reemergence of virus with subsequent graft loss, the use of hepatitis B immunoglobulin (HBIG) and nucleot(s)ide analogs have revolutionized the field and survival statistics now equal or better those seen for other indications $[3,4]$.

While such therapies are effective they remain prohibitively expensive, and resistance with subsequent disease emergence is a constant consideration. In principle, HBV surface antigen (sAg) seroconversion with adequate surface antibody titer response, either spontaneously or by vaccination, can be effective at preventing recurrence of disease in the donor organ [5]. However, in practice, development of an adequate HB surface antibody $(\mathrm{sAb})$ titer in such individuals has been difficult

\footnotetext{
"Corresponding author.
}

using standard or even double-dose intra-muscular (IM) vaccination regimens [6,7]. A recent study reported response rates only of approximately $8 \%$ with a double-dose regimen [8].

Several case reports and small studies have shown that in patients with HBsAg seroconversion, and with adequate titer, pre- or post-solid organ transplantation, that the emergence of HBV infection can be largely prevented [9-11]. Herein, we present a HBV related liver transplant recipient who underwent intra-dermal (ID) HBV vaccination after failing to respond to a standard IM vaccination course. She developed a robust immunologic response that enabled her to discontinue her antiviral medications.

\section{Case Report}

A 61-year-old Korean female with a history of HBV end-stage liver disease presented for evaluation. She likely acquired the virus vertically as all other risk-factors for HBV acquisition were negative. Initial serologic evaluation showed the following: HBV viral load was 4 million IU $/ \mathrm{ml}$; serologies were $\mathrm{HB}$ envelope antigen $(\mathrm{eAg})^{+}, \mathrm{HBsAg}+, \mathrm{HB}$ core antibody $(\mathrm{cAb})^{+}, \mathrm{HB}$ surface antibody ( $(\mathrm{AAb})-$, and transaminases were appro-ximately 1.5 times the upper limit of normal with normal total 
bilirubin and alkaline phosphatase levels. The remainder of her concomitant liver disease work-up was negative. Her radiologic work-up showed a relatively small liver with no masses, and also noted splenomegaly. Her medical history was otherwise significant for hypercholesterolemia and thrombocytopenia.

She was started on lamivudine (LAM) and achieved a virus undetectable state within 6 months. One year later, she was found to have re-emergence of virus with a viral load of $60,000 \mathrm{IU} / \mathrm{ml}$; development of LAM resistant virus was suspected. Famciclovir was added with adequate viral suppression. Over the next 2 years, she developed multiple episodes of liver decompensation manifested as hepatic encephalopathy, a volume-overload state and numerous infections. Ultimately, she underwent OLT in 2002. Her post-operative course was uncomplicated while utilizing an immunosuppressive regimen consisting of tacrolimus and prednisone, the latter being tapered off after 6 months. She initially received HBIG $0.06 \mathrm{ml} / \mathrm{kg}$ intramuscular (IM) and was on combination therapy with LAM/Adefovir (ADV) with an undetectable virus load. In 2008, her viral serologies consisted of the following: HBV-DNA-viral load, $\mathrm{HBcAb}+, \mathrm{HBeAg}^{-}$, $\mathrm{HBeAb}+, \mathrm{HBsAg}^{-}, \mathrm{HBsAb}-$, with normal transaminases, total bilirubin and alkaline phosphatase levels. During this interval, the patient intermittently stopped her antiviral and immunosuppressant medications due to financial reasons.

In 2009, in order to prevent re-emergence of virus, HBV vaccination with a standard IM dose and subsequently a double dose IM regimen were attempted — both being unsuccessful at eliciting an immune response, as defined by a HBsAb titer $>10 \mathrm{mIU} / \mathrm{mL}$. Therefore, she was given a $40 \mu \mathrm{g}$ ID HBV vaccine split into 2 aliquots (20 $\mu \mathrm{g}$ in each arm). The patient experienced transient erythema and localized tenderness at the sites of the injection with no other systemic side effects. Laboratory evaluation at three months post ID vaccination showed a robust $\mathrm{HBsAb}$ response with an antibody titer of 143 $\mathrm{mIU} / \mathrm{ml}$. The titer and its durability were reconfirmed six months post ID vaccination and her LAM/ADV therapy was discontinued. Her liver injury tests have remained at baseline with no detectable HBV DNA and persistent HBsAb titers with multiple sampling. Currently, her $\mathrm{HBsAb}$ titers are scheduled to be checked every three months with a booster (IM or ID) dose of vaccine if the $\mathrm{HBsAb}$ titer is found to be less than $100 \mathrm{mIU} / \mathrm{ml}$.

\section{Discussion}

The use of HBIG and nucleot(s)ide analogs have revolutionized the field of HBV treatment after OLT. While extremely effective at preventing re-infection of the graft, lifelong therapy is required using a combination of HBIG and/or nucleot(s)ide analogs [12]. This represents a significant financial burden to the health care system. One year of HBIG typically can cost up to 100,000 dollars and nucleot(s)ide therapy costs can exceed one to two thousand dollars monthly [13]. Furthermore, non-compliance can promote the development of viral resistance and re-emergence of the infection with subsequent graft loss. Several studies have suggested that if an adequate $\mathrm{HBsAb}$ titer can be achieved via vaccination either prior to or post-OLT, re-infection rates are negligible $[4,6]$. The cost-benefit to the healthcare system would thus be substantial.

In immunodebilitated patients, such as those with chronic liver disease, attempts at eliciting an adequate immune response via IM standard, double-dose or acelerated schedule vaccination has been largely unsuccessful [14]. Recently, ID vaccination has been shown to be an effective mode of vaccination for those individuals, who are refractory to standard IM dosing [15]. The rationale for the ID vaccination rests upon the principle that there are a large number of dendritic cells present in the dermis which can enhance antigen presentation to immune effector cells. Thus, it has been hypothesized that ID vaccination might elicit a better immune response than the IM route while maintaining a similar safety record.

This concept of ID immunogenicity has already been utilized successfully with influenza or rabies vaccines where antibody response rates have been shown to be higher even when using fractional dosing [16]. Furthermore, in renal transplant patients who did not have any evidence of HBV exposure but were refractory to IM vaccination, this strategy produced a robust response in $62.5 \%$ of patients [17].

An extension of this idea, in chronic liver disease, however was not supported in a small study by Angelico et al. who found that HBV OLT patients who were $\mathrm{HBsAg}-$ and refractory to IM vaccination did not respond to subsequent ID administration [18]. This study however used a small, likely suboptimal, dose $(10 \mu \mathrm{g})$ of vaccine, the patients had received HBIG immediately prior to the study, had detectable HBsAb at the time of enrollment, and all were on cyclosporine. The $\mathrm{HBsAg}-$ status was more likely due to the neutralizing effect of HBIG derived HBsAb rather than a low or non-replicative state of the HBV.

This patient remained on combination antiviral (LAM/ ADV) regimen, suggesting effective suppression with minimal if any viral replication as evidenced by negative HBV-DNA and HBsAg status. After this patient failed standard HBV IM dosing, ID vaccination was initiated. The rationale being that she was on minimal immunosuppression, she had spontaneously lost HBsAg and was at significant risk for recurrence of HBV in light of her 
poor history of compliance with her antiviral regimen. As such, she underwent ID vaccination and had a robust immunologic response after a single dose. The titer, as well as its durability, was confirmed at three and six months thereafter. As a result, her antiviral regimen was discontinued with close follow-up.

ID vaccination elicits a significant immunologic response and may represent an important therapeutic modality to prevent re-emergence of $\mathrm{HBV}$ in select individuals who are HBV-DNA and HBsAg negative. If validated, this may represent an extremely cost-effective strategy in the long-term care of a subset of immunosuppressed patients who are $\mathrm{HBcAb}+, \mathrm{HBsAg}-$ and have undergone OLT.

Future prospective studies are needed to evaluate the most effective dose considering cost, and the achieved level and durability of the immune response. Given the documented success of ID vaccination, it would appear as reasonable option in patients refractory to prior standard or high dose IM HBV vaccination.

\section{REFERENCES}

[1] C. W. Shepard, E. P. Simard, L. Finelli, A. E. Fiore and B. P. Bell, "Hepatitis B Virus Infection: Epidemiology and Vaccination," Epidemiologic Reviews, Vol. 28, No. 1, 2006, pp. 112-125. doi:10.1093/epirev/mxj009

[2] A. R. Zanetti, P. Van Damme and D. S. Vaccine, "The Global Impact of Vaccination against Hepatitis B: A Historical Overview," Vaccine, Vol. 26, No. 49, 2008, pp. 6266-6273. doi:10.1016/j.vaccine.2008.09.056

[3] N. A. Terrault and T. L. Wright, "Hepatitis B Virus Infection and Liver Transplantation," Gut, Vol. 40, No. 5, 1997 , pp. 568-571.

[4] J. P. Lerut, M. Donataccio, O. Ciccarelli, et al., "Liver Transplantation and HBsAg-Positive Postnecrotic Cirrhosis: Adequate Immunoprophylaxis and Delta Virus CoInfection as the Significant Determinants of Long-Term Prognosis," Journal of Hepatology, Vol. 30, No. 4, 1999, pp. 706-714. doi:10.1016/S0168-8278(99)80203-7

[5] M. Wiedmann, U. G. Liebert, U. Oesen, H. Porst, M. Wiese, S. Schroeder, U. Halm, J. Mössner and F. Berr, "Decreased Immunogenicity of Recombinant Hepatitis B Vaccine in Chronic Hepatitis C. Hepatology," Vol. 31, No. 1, 2000, pp. 230-234. doi:10.1002/hep.510310134

[6] U. Bienzle, M. Günther, R. Neuhaus, P. Vandepapeliere, J. Vollmar, A. Lun and P. Neuhaus, "Immunization with an Adjuvant Hepatitis B Vaccine after Liver Transplantation for Hepatitis B-Related Disease," Hepatology, Vol. 38, No. 4, 2003, pp. 811-819.

[7] M. Arslan, R. H. Wiesner, C. Sievers, K. Egan, N. N. Zein, "Double-Dose Accelerated Hepatitis B Vaccine in Patients with End-Stage Liver Disease," Liver Transplantation, Vol. 7, No. 4, 2001, pp. 314-320. doi:10.1053/jlts.2001.23069

[8] C. M. Lo, C. L. Liu, S. C. Chan, G. K. Lau and S. T. Fan,
"Failure of Hepatitis B Vaccination in Patients Receiving Lamivudine Prophylaxis after Liver Transplantation for Chronic Hepatitis B," Journal of Hepatology, Vol. 43, No. 2, 2005, pp. 283-287. doi:10.1016/j.jhep.2005.03.013

[9] F. Fabrizi, V. Dixit, P. Messa and P. Martin, "Intradermal vs Intramuscular Vaccine against Hepatitis B Infection in Dialysis Patients: A Meta-Analysis of Randomized Trials," Journal of Viral Hepatitis, Vol. 18, No. 10, 2010, pp. 730-737.

[10] A. Aziz, S. Aziz, D. S. Li, L. Murphy, N. Leone, M. Kennedy, S. Dhillon and D. H. Van Thiel, "Efficacy of Repeated High-Dose Hepatitis B Vaccine $(80 \mu \mathrm{g})$ in Patients with Chronic Liver Disease," Journal of Viral Hepatitis, Vol. 13, No. 4, 2006, pp. 217-221. doi:10.1111/j.1365-2893.2005.00674.x

[11] L. Sangaré, L. Manhart, D. Zehrung and C. C. Wang, "Intradermal Hepatitis B Vaccination: A Systematic Review and Meta-Analysis," Vaccine, Vol. 27, No. 12, 2009, pp. 1777-1786. doi:10.1016/j.vaccine.2009.01.043

[12] R. P. Perrillo, M. Kruger, T. Sievers and J. R. Lake, "Posttransplantation: Emerging and Future Therapies," Seminars in Liver Disease, Vol. 20, No. S1, 2000, PP. 13-17.

[13] F. Bihl, S. Russmann, V. Gurtner, L. Di Giammarino, L. Pizzi-Bosman, M. Michel, A. Cerny, A. Hadengue, P. Majno, E. Giostra, D. Castelli and G. Mentha, "Hyperimmune Anti-HBs Plasma as Alternative to Commercial Immunoglobulins for Prevention of HBV Recurrence after Liver Transplantation," BMC Gastroenterology, Vol. 4, No. 71, 2010. doi:10.1186/1471-230X-10-71

[14] E. A. Arbizu, R. B. Marugán, J. Y. Grijalba, P. L.Serrano and L. G. Grande and S. D. C. Terrón, "Intramuscular versus Intradermal Administration of Anti-Hepatitis B Vaccine in Non-Cirrhotic Hepatitis C Patients," Vaccine, Vol. 21, No. 21-22, 2003, pp. 2747-2750. doi:10.1016/S0264-410X(03)00221-4

[15] S. Dhillon, C. Moore, S. D. Li, A. Aziz, A. Kakar, A. Dosanjh, A. Beesla, L. Murphy and D. H. Van Thiel, "Efficacy of High-Dose Intra-Dermal Hepatitis B Virus Vaccine in Previous Vaccination Non-Responders with Chronic Liver Disease," Digestive Diseases and Sciences, Vol. 57, No. 1, 2012, pp. 215-220. doi:10.1007/s10620-011-1996-0

[16] R. B. Belshe, F. K. Newman, J. Cannon, C. Duane, J. Treanor, C. Van Hoecke, B. J. Howe and G. Dubin, "Serum Antibody Responses after Intradermal Vaccination against Influenza," The New England Journal of Medicine, No. 351, No. 22, 2004, pp. 2286-2294. doi:10.1056/NEJMoa043555

[17] B. Y. Choy, J. S. Peiris, T. M. Chan, S. K. Lo, S. L. Lui and K. N. Lai, "Immunogenicity of Intradermal Hepatitis B Vaccination in Renal Transplant Recipients," American Journal of Transplantation, Vol. 2, No. 10, 2002, pp. 965-969. doi:10.1034/j.1600-6143.2002.21014.x

[18] M. Angelico, D. Di Paolo, M. O. Trinito, A. Pterolati, A. Araco, S. Zazza, R. Lionetti, C. U. Casciani and G. Tisone, "Failure of a Reinforced Triple Course of Hepatitis B Vaccination in Patients Transplanted for HBV-Related Cirrhosis," Hepatology, Vol. 35, No. 1, 2002, pp. 176-181. 